\title{
Remarks on Band Matrices
}

\author{
L. Elsner and R. M. RedhefFer
}

Received August 15, 1966

Abstract. In this note we consider band- or tridiagonal-matrices of order $k$, whose elements above, on, and below the diagonal are denoted by $b_{i}, a_{i}, c_{i}$. In the periodic case, i.e. $b_{i+m}=b_{\imath}$ etc., we derive for $k=n m$ and $k=n m-1$ formulas for the characteristic polynomial and the eigenvectors under the assumption that $\prod_{i=1}^{m} c_{i} b_{i}>0$. In the latter case it is shown that the characteristic polynomial is divisible by the $m-1$-th minor, as was already observed by Rózsa. We also give estimations for the number of real roots and an application to Fibonacci numbers.

\section{Introduction}

Throughout this note $a_{i}, b_{i}$ and $c_{i}$ for $i=1,2, \ldots, m$ are complex numbers with

$$
b=b_{1} b_{2} \ldots b_{m}, \quad c=c_{1} c_{2} \ldots c_{m}, \quad x^{2}=b c \neq 0 .
$$

The letter $B=B^{m}$ denotes a matrix of $m$ rows and $m+2$ columns,

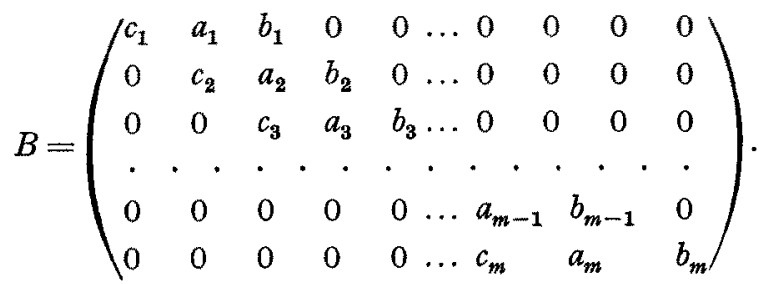

The square $m$ by $m$ matrix obtained from $B$ when the first and last columns are deleted is called a band matrix and is denoted by $B^{\prime}$. The square $m-1$ by $m-1$ matrix obtained from $B^{\prime}$ when the bottom row and right-hand column are deleted, for $m>1$, is denoted by $B^{\prime \prime}$.

Using a square $m$ by $m$ zero matrix $0_{m}$ with $B^{m}$ we can form a matrix $B_{n}=B_{n}^{m}$ of $n m$ rows and $n m+2$ columns,

$$
B_{n}=\left(\begin{array}{ccccc}
B^{m} & 0_{m} & 0_{m} & \ldots & 0_{m} \\
0_{m} & B^{m} & 0_{m} & \ldots & 0_{m} \\
0_{m} & 0_{m} & B^{m} & \ldots & 0_{m} \\
\cdot & \cdot & \cdot & \cdot & \cdot \\
0_{m} & 0_{m} & 0_{m} & \ldots & B^{m}
\end{array}\right)
$$


The square $m n$ by $m n$ matrix obtained from $B_{n}$ when the first and last columns are deleted is called a periodic band matrix and is denoted by $B_{n}^{\prime}$. If the bottom row and last column of $B_{n}^{\prime}$ are deleted the resulting $m n-1$ by $m n-1$ matrix is also called a periodic band matrix and is denoted by $B_{n}^{\prime \prime}$. In justification of this terminology it should be observed that $B_{n}$ has the same form as $B$, except that $m$ has been replaced by $m n$ and the elements are periodic. That is, if the elements of $B_{n}$ are denoted by $a_{i}, b_{i}$ and $c_{i}$ with $1 \leqq i \leqq m n$ then

$$
a_{i+m}=a_{i}, \quad b_{i+m}=b_{i}, \quad c_{i+m}=c_{i} .
$$

As far as we know Rózsa and Lovass-NAGY of Budapest were the first to make a systematic study of periodic band matrices with $m>1$, and in particular [2] and [3] contain the complete solution for $m=2$. Our interest in the subject stems from a lecture which RózsA held at the University of Hamburg in the summer semester, 1966. This lecture was devoted to the proof of the following theorem, which generalizes the result [3] to the case of arbitrary $m$ :

Theorem A (RozSA). Let determinants $D_{m}=D_{m}(\lambda)$ be defined by

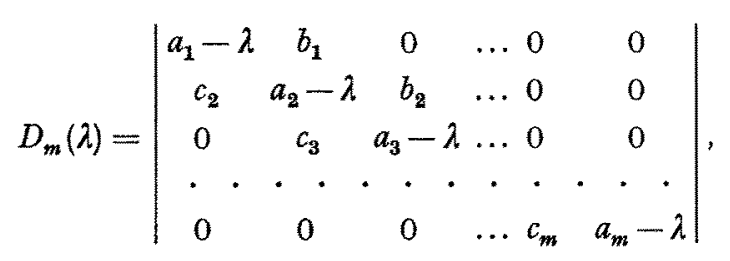

and let $D_{m}^{*}(\lambda)$ denote the determinant obtained from $D_{m}(\lambda)$ when the first row and column are removed. Then, if $B_{n}^{\prime \prime}$ is symmetric, the characteristic values of $B_{n}^{\prime \prime}$ are the values $\lambda$ for which $D_{m-1}(\lambda)=0$ or for which

$$
D_{m}(\lambda)-b_{m}^{2} D_{m-1}^{*}(\lambda)=2 b_{1} b_{2} \ldots b_{m} \cos \frac{k \pi}{n}, \quad k=1,2, \ldots, n-1 .
$$

Our reasons for writing are threefold. First, our method is wholly elementary, while Rózsa used a number of advanced tools from the algebra of matrices and determinants. Second, we obtain results for $B_{n}^{\prime}$ as well as $B_{n}^{\prime \prime}$, while RózsA was able, he said, to get results in the case of arbitrary $m$ for $B_{n}^{\prime \prime}$ only. Third, we treat the general case while RozsA's analysis applies only to the symmetric case

$$
c_{1}=b_{m}, c_{2}=b_{1}, \ldots, c_{m}=b_{m-1} \text {. }
$$

In particular, we shall find that RózsA's result continues to hold in the asymmetric case, if the factor $b_{m}^{2}$ on the left is replaced by $b_{m} c_{1}$ and the factor $b_{1} b_{2} \ldots b_{m}$ on the right is replaced by $x$.

\section{The Recursive Solution}

Let $x^{\prime}$ be an $m$-dimensional column vector with components $\left(x_{1}, x_{2}, \ldots, x_{m}\right)$ and let $x$ be an $(m+2)$-dimensional column vector with components

$$
\left(x_{0}, x_{1}, \ldots, x_{m}, x_{m+1}\right) \text {. }
$$

Evidently $\lambda$ is a characteristic value for $B^{\prime}$ if and only if the system $B^{\prime} x^{\prime}=\lambda x^{\prime}$ has a nontrivial solution $x^{\prime}$. Since $x_{1}=0$ implies $x^{\prime}=0$ we can suppose that $x_{1}=1$. 
The system $B^{\prime} x^{\prime}=\lambda x^{\prime}$ has an unsymmetric structure because two columns were deleted in passing from $B$ to $B^{\prime}$. But the system $B x=\lambda x^{\prime}$ has a very symmetric structure; it is

$$
c_{k} x_{k-1}+\left(a_{k}-\lambda\right) x_{k}+b_{k} x_{k+1}=0 \quad(k=1,2, \ldots, m) .
$$

If, now, we require $x_{0}=x_{m+1}=0$ the resulting system is identical with $B^{\prime} x^{\prime}=\lambda x^{\prime}$. In other words $\lambda$ is a characteristic value for $B^{\prime}$ if and only if the system (1) has a solution with $x_{0}=x_{m+1}=0$ and $x_{1}=1$. Replacing $m$ by $m-1$ in this observation, we see that $\lambda$ is a characteristic value for $B^{\prime \prime}$ if and only if (1) has a solution with $x_{0}=x_{m}=0$ and $x_{1}=1$.

If the $k$-th equation (1) is solved for $x_{k+1}$ and the result is used together with $x_{k}=x_{k}$ we get a recurrence formula,

$$
\left(\begin{array}{c}
x_{k} \\
x_{k+1}
\end{array}\right)=\frac{1}{b_{k}}\left(\begin{array}{cc}
0 & b_{k} \\
-c_{k} & \lambda-a_{k}
\end{array}\right)\left(\begin{array}{c}
x_{k-1} \\
x_{k}
\end{array}\right) \text {. }
$$

This is solved by means of the products

$$
\left(\begin{array}{ll}
p_{k} & q_{k} \\
r_{k} & s_{k}
\end{array}\right)=\left(\begin{array}{cc}
0 & b_{k} \\
-c_{k} & \lambda-a_{k}
\end{array}\right) \cdots\left(\begin{array}{cc}
0 & b_{2} \\
-c_{2} & \lambda-a_{2}
\end{array}\right)\left(\begin{array}{cc}
0 & b_{1} \\
-c_{1} & \lambda-a_{1}
\end{array}\right)
$$

where the polynomials $p_{k}=p_{k}(\lambda)$, and so on, are defined by the equation. For brevity we denote this product by $\prod_{k}(\lambda), 1 \leqq k \leqq m$. Since

$$
\left(\begin{array}{c}
x_{k} \\
x_{k+1}
\end{array}\right)=\frac{1}{b_{k} b_{k-1} \cdots b_{2} b_{1}}\left(\begin{array}{cc}
p_{k} & q_{k} \\
r_{k} & s_{k}
\end{array}\right)\left(\begin{array}{l}
0 \\
1
\end{array}\right)
$$

the values of $x_{k}$ and the desired condition $x_{m}=0$ or $x_{m+1}=0$ can be expressed with ease.

We write the characteristic polynomial of $B_{n}^{\prime}$ or $B_{n}^{\prime \prime}$ as

$$
\lambda^{m n}+\cdots \text { or } \lambda^{m n-1}+\cdots
$$

so that the leading coefficient is 1 . The leading term of $p_{m}(\lambda), q_{m}(\lambda), r_{m}(\lambda)$ or $s_{m}(\lambda)$ is

$$
-b_{m} c_{1} \lambda^{m-2}, \quad b_{m} \lambda^{m-1}, \quad-c_{1} \lambda^{m-1}, \quad \lambda^{m}
$$

respectively, as is easily proved by induction. Since the characteristic polynomial is wholly determined by the characteristic roots, we can summarize our analysis as follows:

Remark 1. Let polynomials $p_{k}, q_{k}, r_{k}, s_{k}$ be defined as above. Then the characteristic polynomial of $B^{\prime}$ or $B^{\prime \prime}$ is $s_{m}(\lambda)$ or $b_{m}^{-1} q_{m}(\lambda)$, respectively. The characteristic vector for $B^{\prime}$ belonging to $\lambda$ is

$$
\left(1, \frac{s_{1}(\lambda)}{b_{1}}, \frac{s_{2}(\lambda)}{b_{1} b_{2}}, \ldots, \frac{s_{m-1}(\lambda)}{b_{1} b_{2} \ldots b_{m-1}}\right)
$$

where $s_{m}(\lambda)=0$, and that for $B^{\prime \prime}$ is the same, with $m-1$ in place of $m$, and with $q_{m}(\lambda)=0$ instead of $s_{m}(\lambda)=0$.

We define now $D_{m}, D_{m}^{*}$ as in Theorem $\mathrm{A}$ and analogously $D_{m-1}, D_{m-1}^{*}$ with $D_{0}=1, D_{0}^{*}=0, D_{1}^{*}=1$ by convention. Then we obtain easily 
Remark 2. The following relations hold for $m \geqq 1$ :

$$
\begin{array}{ll}
p_{m}(\lambda)=(-)^{m-1} b_{m} c_{1} D_{m-1}^{*}(\lambda), & q_{m}(\lambda)=(-)^{m-1} b_{m} D_{m-1}(\lambda), \\
r_{m}(\lambda)=(-)^{m} c_{1} D_{m}^{*}(\lambda), & s_{m}(\lambda)=(-)^{m} D_{m}(\lambda) .
\end{array}
$$

For proof, form $B^{*}$ by deleting the first row and column of $B$. Apply Remark 1 to the corresponding product $\prod_{m}^{*}$ as well as to $\prod_{m}$ and note that $\prod_{m}^{*}$ and $\prod_{m}$ are simply related.

We introduce

\section{The Roots in the Periodic Case}

$$
A=A(\lambda)=x^{-1} \prod_{m}(\lambda) \text {. }
$$

Since det $\prod_{m}(\lambda)=x^{2}$, we have $\operatorname{det} A=1$. The trace of $A$ is $2 t$, where $t=t(\lambda)$ is defined by

$$
2 x t(\lambda)=p_{m}(\lambda)+s_{m}(\lambda)
$$

We have by the Cayley-Hamilton theorem

$$
A^{2}-2 t A+I=0 \quad I=\text { identity-matrix }
$$

and hence with $M_{n}=A^{n}$

$$
M_{n+1}+M_{n-1}=2 t M_{n} .
$$

Setting $t=\cos \theta$, where $\theta$ is a real or complex angle, we see that the solution of the difference equation (3) is given by

$$
M_{n}=M_{1} \frac{\sin n \theta}{\sin \theta}-M_{0} \frac{\sin (n-1) \theta}{\sin \theta} .
$$

(Here and elsewhere, $\sin k \theta / \sin \theta$ is to be replaced by its limit, $k \cos k \theta / \cos \theta$, if $\sin \theta=0$.) It follows, in particular, that

$$
A^{n}=A \frac{\sin n \theta}{\sin \theta}-I \frac{\sin (n-1) \theta}{\sin \theta} .
$$

Upon applying Remark 1 to $B_{n}$ instead of $B$ we obtain

Remark 3. The characteristic roots of $B_{n}^{\prime}$ are the values $\lambda$ for which simultaneously

$$
2 \varkappa \cos \theta=p_{m}(\lambda)+s_{m}(\lambda) \text { and } s_{m}(\lambda) \frac{\sin n \theta}{\sin \theta}=\varkappa \frac{\sin (n-1) \theta}{\sin \theta} \text {. }
$$

The characteristic roots of $B_{n}^{\prime \prime}$ are the values $\lambda$ for which

$$
q_{m}(\lambda)=0 \quad \text { or } \quad p_{m}(\lambda)+s_{m}(\lambda)=2 x \cos \frac{k \pi}{n}, \quad k=1,2, \ldots, n-1 .
$$

Remark 2 gives a corresponding version of Remark 3 with $p_{m}, q_{m}$ and $s_{m}$ replaced by appropriate subdeterminants of $D_{m}$. The part of Remark 3 pertaining to $B_{n}^{\prime \prime}$ thus gives the generalized version of RozsA's theorem mentioned in the introduction. Conversely, our result for $B_{n}^{\prime \prime}$ can be deduced from RózsA's by an affine transformation. 


\section{Characteristic Vectors}

To discuss the characteristic vectors $x^{\prime}$ for $B_{n}^{\prime}$ and $x^{\prime \prime}$ for $B_{n}^{\prime \prime}$ it is convenient to denote the elements of $B_{n}$ by $a_{i}, b_{i}$ and $c_{i}$ with $1 \leqq i \leqq m n$ and with the periodicity condition. The products $\prod_{i}=\prod_{i}(\lambda)$ for $i>m$ are interpreted accordingly. Any index $i \geqq 1$ can be written $i=m j+l$ with $1 \leqq l \leqq m$. Then

$$
b_{1} b_{2} \ldots b_{i}=b^{i} b_{1} b_{2} \ldots b_{l} \text { and } \prod_{i}=\prod_{l}\left(\prod_{m}\right)^{i}
$$

Since $\prod_{m}=x A$ the formula (4) for $A^{n}$ gives a similar formula for $\left(\prod_{m}\right)^{j}$. Upon applying Remark 1 to $B_{n}$ instead of $B$ we get:

Remark 4. Let $\theta=\theta(\lambda)$ be defined by

$$
2 \varkappa \cos \theta=p_{m}(\lambda)+s_{m}(\lambda)
$$

where $\lambda$ is a characteristic value for $B_{n}^{\prime}$ or $B_{n}^{\prime \prime}$, as the case may be. Let the corresponding characteristic vector be $\left(x_{i}\right)$ with $1 \leqq i \leqq m n$ or $1 \leqq i \leqq m n-1$, respectively. Then for $j=0,1,2, \ldots$ and $1 \leqq l \leqq m$ we have

where $\varrho=\sqrt{c / b}$.

$$
x_{m+l}=\varrho^{j-1} \frac{\sin j \theta}{\sin \theta} x_{m+l}-\varrho^{j} \frac{\sin (j-1) \theta}{\sin \theta} x_{l}
$$

The interest of the result is that it gives $x_{i}$ for all $i$ as soon as $\lambda$ and the initial values

$$
x_{1}, x_{2}, \ldots, x_{2 m}
$$

are known. These initial values can be found by the formula of Remark 1 or by recursive solution of (1). Of course the recursive solution gives $x_{i}$ for all $i$, but if $i$ is large, the formula of Remark 4 is simpler and more accurate.

\section{The Characteristic Polynomial}

The polynomial of degree $n$ defined by

$$
U_{n}(\cos \theta)=\frac{\sin (n+1) \theta}{\sin \theta}
$$

is called the Chebychev polynomial of the second kind [1]. In terms of $U_{n}$ the result (4) reads

$$
A^{n}=A U_{n-1}(t)-I U_{n-2}(t)
$$

Applying Remark 1 to $B_{n}^{\prime}$ and $B_{n}^{\prime \prime}$, using (6) and the relation

we obtain

$$
U_{n+1}(t)+U_{n-1}(t)=2 t U_{n}(t)
$$

Remark 5. Let $t=t(\lambda)$ be defined by $2 x t=p_{m}(\lambda)+s_{m}(\lambda)$. Then the characteristic polynomial of $B_{n}^{\prime}$ is

$$
x^{n} U_{n}(t)-p_{m}(\lambda) x^{n-1} U_{n-1}(t)
$$


and the characteristic polynomial of $B_{n}^{\prime \prime}$ is

$$
x^{n-1} s_{m-1}(\lambda) U_{n-1}(t)
$$

under the convention that $s_{0}(\lambda)=1$.

Remark 5 gives identities similar to those of Remark 2 for $B_{n}^{\prime}$ and $B_{n}^{\prime \prime}$. In particular, setting $\lambda=0$ we get the constant term of the characteristic equation,

$$
(-)^{m n} \operatorname{det} B_{n}^{\prime} \quad \text { or }(-)^{m n-1} \operatorname{det} B_{n}^{\prime \prime} \text {. }
$$

Since the choice $\theta=\pi / 2$ in (5) gives $U_{n}(0)$ we are led to the following as a special case: Suppose

Then

$$
p_{m}(0)+s_{m}(0)=0
$$

$$
(-)^{m n} \operatorname{det} B_{n}^{\prime}=x^{n}, \quad-p_{m}(0) x^{n-1}, \quad-x^{n}, \quad p_{m}(0) \varkappa^{n-1}
$$

according as $n \equiv 0,1,2$ or $3 \bmod (4)$, respectively. In the same circumstances the respective values for $\operatorname{det} B_{n}^{\prime \prime}$ are given by

$$
(-)^{m n} \operatorname{det} B_{n}^{\prime \prime}=0, \quad-s_{m-1}(0) x^{n-1}, \quad 0, \quad s_{m-1}(0) x^{n-1}
$$

\section{Conditions for Real Roots}

In this section we give conditions under which $B_{n}^{\prime}$ and $B_{n}^{\prime \prime}$ have at least $m n$ const distinct real roots as $n \rightarrow \infty$. It is convenient to assume that $x$ is real and positive, and of course, that $a_{i}, b_{i}$ and $c_{i}$ are real.

The desired results can be read off from a plot of $y=t(\lambda)$ together with the horizontal lines

$$
y=\cos \frac{k \pi}{n}, \quad k=1,2, \ldots, n-1 .
$$

Such a plot might have the appearance suggested by the figure when $m=5$ and $n=10$. Each intersection point gives a real root of $B_{n}^{\prime \prime}$.

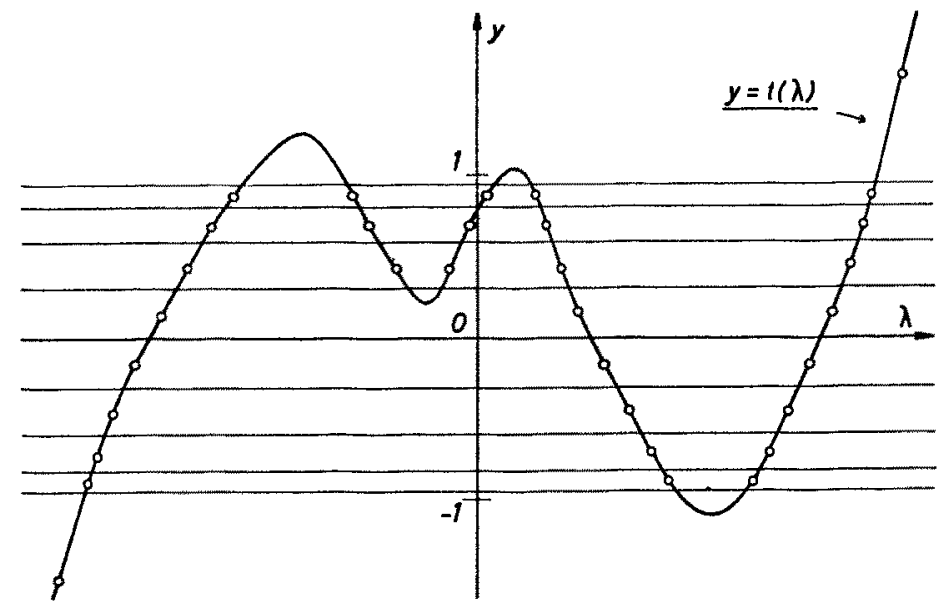

Fig. 1 
To discuss the corresponding graphical interpretation for $B_{n}^{\prime}$ we note that a plot of

$$
y=\frac{U_{n}(t)}{U_{n-1}(t)}
$$

vs. $t$ consists of $n$ continuous curves, in each of which $y$ ranges from $-\infty$ to $\infty$. The equation

$$
p_{m}(\lambda)=\varkappa \frac{U_{n}(t)}{U_{n-1}(t)}
$$

given by Remark 5 therefore has at least one real root in each interval on which the graph of $t(\lambda)$ joins one line (8) to an adjacent one, and also in each of the two unbounded intervals. These portions of the graph are indicated by the round dots in the figure.

We now introduce the following definition:

Definition. A real polynomial $P(x)$ of degree $n$ is of Chebychev type if there are $n+1$ values $x_{i}$ such that

$$
x_{0}>x_{1}>x_{2} \ldots>x_{n} \text { and }(-)^{k} P\left(x_{k}\right) \geqq 1 \quad(k=0,1, \ldots, n) .
$$

Evidently, a Chebychev polynomial is an extremal polynomial of this class, in several senses of the word "extremal".

By the Lagrange interpolation formula we see that a polynomial is of Chebychev type if and only if it admits the representation

where

$$
P(x)=\sum_{k=0}^{n} \frac{Q(x)}{x-x_{k}} \frac{(-)^{k} A_{k}}{Q^{\prime}\left(x_{k}\right)}
$$

$$
Q(x)=\left(x-x_{0}\right)\left(x-x_{1}\right) \ldots\left(x-x_{n}\right), \quad A_{k} \geqq 1,
$$

and $x_{k}$ are as in the definition. If $P(x)$ admits such a representation then it admits one in which the values $x_{1}, x_{2}, \ldots, x_{n-1}$ are the roots of $P^{\prime}(x)=0$, and in which furthermore

$$
P\left(x_{0}\right)=(-)^{n} P\left(x_{n}\right)=1 \text {. }
$$

The latter representation is unique. Other characterizations can be obtained by writing

$$
\left.P^{\prime}(x)=\text { const }\right)\left(x-x_{1}\right)\left(x-x_{2}\right) \ldots\left(x-x_{n-1}\right)
$$

and integrating to get $P(x)$.

The graphical interpretation of the foregoing discussion leads to the following:

Remark 6. Let $a_{i}, b_{i}, c_{i}$ and $x=\sqrt{b c}$ be real, with $x>0$. Let a polynomial $t(\lambda)$ be defined by

$$
2 \varkappa t(\lambda)=p_{m}(\lambda)+s_{m}(\lambda) .
$$

Then if $t(\lambda)$ is a polynomial of Chebychev type the matrices $B_{n}^{\prime}$ and $B_{n}^{\prime \prime}$ have at least

$$
m n-2 m+2 \text { and } m n-m
$$

distinct real characteristic values, respectively. But if $t(\lambda)$ is not a polynomial of Chebychev type there is a constant $m_{1}<m$ such that the number of real characteristic roots of $B_{n}^{\prime}$ or $B_{n}^{\prime \prime}$, counting multiplicity, does not exceed $m_{1} n$ for sufficiently large $n$. 
It should be observed that the derivation of Remark 6 not only gives somewhat more information about the number of real roots than is there stated, but also gives quite detailed information about their location.

So far we have assumed $b c>0$. If $b c<0$ Remark 3 shows that the only real roots of $B_{n}^{\prime \prime}$ are among the roots of $q_{m}(\lambda)$ or $p_{m}(\lambda)+s_{m}(\lambda)$ and hence, there are at most $2 m-1$ real roots. The corresponding question for $B_{n}^{\prime}$ is left as an open problem.

An evident consequence of Remark 6 is:

Remark 7. If the products $c_{1} b_{m}, c_{2} b_{1}, c_{3} b_{2}, \ldots, c_{m} b_{m-1}$ are all positive, then $t(\lambda)$ is a polynomial of Chebychev type.

\section{An Application to Number Theory}

The Fibonacci numbers $0,1,1,2,3,5, \ldots$ are defined by

$$
f_{0}=0, \quad f_{1}=1, \quad f_{n+1}=f_{n}+f_{n-1}, \quad n \geqq 1 .
$$

It was pointed out by CoLLATz that Remark 5 gives an extremely simple proof of the following well-known theorem:

Remark 8 (e.g. [4], p. 148). If $k$ is divisible by $m$, then $f_{k}$ is divisible by $f_{m}$.

Collatz's proof, presented here by permission, is as follows. By induction, we obtain the well-known formula

$$
f_{j+1}=D_{j} \text { with } D_{j}=\left|\begin{array}{cccc}
1 & -1 & & \\
1 & 1 & \ddots & 0 \\
1 & \ddots & \\
0 & \ddots & -1 \\
0 & & 1
\end{array}\right|{ }_{j}
$$

The assumption that $m$ divides $k$ gives

for an integer $n$.

$$
k-1=(n-1) m+m-1
$$

Now, if we regard $D_{k-1}$ as the determinant of a periodic band-matrix with period $m$, Remark 5 with $\lambda=0$ gives

where

$$
D_{k-1}=K^{n-1} s_{m-1}(0) U_{n-1}(l)
$$

$$
K=(i)^{m-1} \text { and } 2 K t=p_{m}(0)+q_{m}(0) .
$$

It is evident that $t$ is a Gaussian integer (that is, $t=\alpha+\beta i$ with $\alpha$ and $\beta$ integers) and the same is true of $U_{n-1}(t)$. Since Remark 2 gives $s_{m-1}(0)=(-)^{m-1} D_{m-1}$, we conclude that

$$
D_{k-1}=D_{m-1} \cdot(\text { Gaussian integer })
$$

The Gaussian integer in the equation is necessarily real, as the $D_{j}^{\text {'s }}$ are real, and thus, the proof is complete. 
Remark 8 can be generalized to sequences of the following types:

respectively

$$
f_{0}=0, \quad f_{1}=1, \quad f_{n+1}=a f_{n}+b^{2} f_{n-1}
$$

$$
f_{0}=0, \quad f_{1}=1, \quad f_{n+1}=a f_{n}-b^{2} f_{n-1}
$$

with integers $a, b$. Observe that $K^{n-1} U_{n-1}(r / K)$ is an integer for $r$ and $K$ integral.

We get analogous results for sequences of polynomials which are recursively defined by

$$
p_{0}=1, \quad p_{1}(\lambda)=a-\lambda, \quad p_{i+1}(\lambda)=(a-\lambda) p_{i}(\lambda)+b p_{i-1}(\lambda)
$$

for arbitrary $a, b$. Here we have: If $i \mid m$, then $p_{i-1}(\lambda) \mid p_{m-1}(\lambda)\left(\right.$ e.g. $\left.p_{n}(\lambda)=U_{n}(\lambda)\right)$.

\section{References}

[1] Abramowitz, M., and I. Stegun: Handbook of mathematical functions, p. 776. New York: Dover Pub. Inc. 1965.

[2] Lovass-NAGY, V., u. P. RózsA: Die Berechnung von Ausgleichvorgängen auf längskompensierten Fernleitungen. Arch. Elektrotech. 49, 260-270 (1964).

[3] - - Matrix analysis of transient voltage distributions in alternating ladder networks. Proc. I.E.E., vol. 110, No. 9, September 1963.

[4] HARDY, G. H., and E.M. WRIGHT: An introduction to the theory of numbers. London: Oxford University Press 1960.

Department of Mathematics

University of California

Los Angeles, California 90024, USA
Institut für Angewandte Mathematik der Universität Hamburg 2000 Hamburg 13

Rothenbaumchaussee $67 / 69$ 